\title{
-NOTES-
}

\section{ON A SPECIAL BOLZA VARIATIONAL PROBLEM, AND THE MINIMIZATION OF SUPERAERODYNAMIC HYPERSONIC NOSE DRAG*}

BY H. S. TAN (Therm-Electric Meters Co., Ithaca, N. Y.)

Instead of a definite integral, let the following expression be given:

$$
D=G\left(y_{0}, y_{l}\right)+\int_{0}^{l} F\left(y, y^{\prime}, x\right) d x,
$$

where $G$ is a known function of $y_{0}$ and $y_{l}, y_{0}$ and $y_{\imath}$ may or may not be specified. It is desired to find the optimum function $y(x)$ that minimizes expression (1). This is a special case of the Bolza problem [1].

The variation of (1) is easily obtained as follows:

$$
\begin{aligned}
\delta D & =D\left(y^{*}\right)-D(y) \\
& =G_{y_{0}} \delta y_{0}+G_{y_{l}} \delta y_{l}+\left[F_{y^{\prime}} \delta y\right]_{0}^{l}+\int_{0}^{l}\left[F_{y}-\left(F_{y^{\prime}}\right)^{\prime}\right] \delta y d x \\
& =\left[G_{y_{0}}-\left(F_{y^{\prime}}\right)_{0}\right] \delta y_{0}+\left[G_{y_{l}}+\left(F_{y^{\prime}}\right)_{l}\right] \delta y_{l}+\int_{0}^{l}\left[F_{\nu}-\left(F_{y^{\prime}}\right)^{\prime}\right] \delta y d x .
\end{aligned}
$$

To insure vanishing of $\delta D$, it is clear that:

(i) throughout the interval $0<x<l$, Euler's variational equation must be satisfied:

$$
\varphi=\left(F_{y}\right)^{\prime}-F_{\nu}=0
$$

(ii) at both ends of the interval, the following end conditions must be met:

$$
\begin{aligned}
& \delta y_{0}=0, \quad \text { or } \quad \psi_{0}=G_{\nu_{0}}-\left(F_{y^{\prime}}\right)_{0}=0, \\
& \delta y_{l}=0, \quad \text { or } \quad \psi_{l}=G_{y_{l}}+\left(F_{\nu^{\prime}}\right)_{l}=0 .
\end{aligned}
$$

The condition of minimization is then furnished by

(iii) the Legendre second variational inequality:

$$
F_{y^{\prime} y^{\prime}}>0, \quad 0<x<l .
$$

Our generalization evidently lies in the inclusion of $G$ term outside the integral, and the corresponding broadened end conditions (4). Indeed, if $G$ is constant, or disappears from (1), then the end conditions simply reduce to the following conventional form for calculus of variations $[2,3,4]$, i.e.

$$
\begin{array}{lll}
\delta y_{0}=0, & \text { or } \quad\left(F_{y^{\prime}}\right)_{0}=0, \\
\delta y_{l}=0, & \text { or } \quad\left(F_{y^{\prime}}\right)_{l}=0 .
\end{array}
$$

* Received June 9, 1958. 
In case

$$
G\left(y_{0}, y_{l}\right)=\int_{0-}^{01} F\left(y, y^{\prime}, x\right) d x+\int_{l-}^{l+} F\left(y, y^{\prime}, x\right) d x,
$$

(1) reduces to following single integral:

$$
D=\int_{0-}^{l+} F\left(y, y^{\prime}, x\right) d x
$$

which is, on the one hand, an immediate extension of the ordinary calculus of variations to include Stieltjes integral at the ends; and on the other hand, a special case of our present formulation.

The present generalization of the variational problem has, in fact, arisen as a result of the search for optimum nose curve that minimizes the superaerodynamic hypersonic drag of an axially symmetric body. The problem is usually so formulated that the nose length $l$ and base radius $y_{\imath}$ are both given, while the tip radius $y_{0}$ may or may not be specified, depending on the situation. By using meridianial coordinates, $x$ axial and $y$ radial, the nose drag has been shown to be given by following integral [5]:

$$
\begin{aligned}
D & =2 \pi \rho V^{2} \int_{0-}^{l}\left\{1+c y^{\prime}\left(1+{y^{\prime}}^{2}\right)^{-1 / 2}\right] y y^{\prime} d x \\
& =\pi \rho V^{2}\left\{y_{l}^{2}+c\left[y_{0}^{2}+2 \int_{0}^{l} y y^{\prime}\left(1+{y^{\prime}}^{2}\right)^{-1 / 2} d x\right]\right\},
\end{aligned}
$$

where $c$ is a constant determined by the ratios of solid surface and gas temperature, and of body and molecular speed. It is easy to see that this drag formula is indeed of type (1), with

$$
G=y_{0}^{2}+\left(y_{l}^{2} / c\right), \quad F=2 y y^{\prime}\left(1+y^{\prime 2}\right)^{-1 / 2}
$$

and end conditions:

$$
\begin{gathered}
\delta y_{\imath}=0 \\
\delta y_{0}=0, \quad \text { or } \quad \psi_{0}=G_{\nu_{0}}-\left(F_{\nu^{\prime}}\right)_{0}=0 .
\end{gathered}
$$

As already pointed out, an optimum solution must be that of Euler's variational equation, which, by putting (9) into (3), takes the following form:

$$
y^{\prime \prime}=-y^{\prime 2}\left(1+{y^{\prime 2}}^{2}\right) / y\left(2-{y^{\prime}}^{2}\right) \text {. }
$$

Its solution, in parametric form, has been obtained, by simple quadrature and use of relations $y^{\prime \prime}=y^{\prime} d y^{\prime} / d y$ and $y^{\prime \prime}=d y^{\prime} / d x$, as follows [6]:

$$
\begin{aligned}
& y=c_{1}\left(1+{y^{\prime 2}}^{2}\right)^{3 / 2} / y^{\prime 2}, \\
& x=\frac{c_{1}}{3}\left\{\frac{2\left(1+y^{\prime 2}\right)^{1 / 2}}{y^{\prime 3}}-\frac{\left(1+y^{\prime 2}\right)^{1 / 2}}{y^{\prime}}+3 \ln \left[\left(1+y^{\prime 2}\right)^{1 / 2}+y^{\prime}\right]\right\}+c_{2} .
\end{aligned}
$$

On putting (9) into (5), the second variational inequality then requires:

$$
F_{y^{\prime} y^{\prime}}=2-y^{\prime 2}>0 \text {, i.e. } y^{\prime}<(2)^{1 / 2}
$$

Differential equation (11) shows there are two branch curves merging at cuspidal 
point $y^{\prime}=(2)^{1 / 2}$; solution (12) shows that the axis $y=0$ can not be reached, and $y$ grows indefinitely both as $y^{\prime}$ approaches zero and infinity. The correct branch of curve is thus specified by requirement (13) which, together with (12), imposes the following limitations on the admissible values of $y^{\prime}$ :

$$
0<y^{\prime}<(2)^{1 / 2}, \quad y^{\prime \prime}<0 .
$$

Now the tip condition $\psi_{0} \delta y_{0}=0$ becomes:

(i) For specified $y_{0}: \delta y_{0}=0$. In this case, in view of the limitations on the admissible values of $y^{\prime}$, it is evident that with prescribed nose length $l$ and base radius $y_{l}$, there is a lower bound for admissible tip radius $y_{0}$, below which no solution exists. Within its admissible range, it is easy to see that specification of $x_{0}, y_{0}, x_{l}, y_{l}$, uniquely determines the four unknowns $C_{1}, C_{2}, y_{0}^{\prime}$ and $y_{\imath}^{\prime}$, by the four independent equations from parametric solution (12).

(ii) For unspecified $y_{0}$ :

$$
\psi_{0}=G_{y_{0}}-\left(F_{y^{\prime}}\right)_{0}=y_{0}\left[1-y_{0}^{\prime}\left(2+y_{0}^{\prime 2}\right)\left(1+y_{0}^{\prime 2}\right)^{-3 / 2}\right]=0 .
$$

Although it is easy to see that both $y_{0}=0$ and $y_{0}^{\prime}=\infty$ are solutions of (15), neither of them can be fulfilled by our solution (12), with finite $l$ and non-vanishing $y_{l}$. However, a plot of $\psi_{0}$ against $y_{0}^{\prime}$ (Fig. 1) shows that the curve crosses axis $\psi_{0}=0$ at $y_{0}^{\prime}=0.7862$ $<(2)^{1 / 2}$ Thus, with unspecified $y_{0}, y_{0}^{\prime}$ can be determined through condition (15). Actual construction of the optimum curve then amounts to specifying $x_{0}, x_{\imath}, y_{0}^{\prime}$ (through $\psi_{0}=0$ ), and $y_{l}$, from which four unknowns $y_{0}, y_{l}^{\prime}, C_{1}, C_{2}$, are determined by four independent equations from parametric solution (12).

It is interesting to note that in this case $y_{0}$ corresponding to $y_{0}^{\prime}=0.7862$ is usually greater than the least admissible value of $y_{0}$ which corresponds to $y_{0}^{\prime}=(2)^{1 / 2}$. This implies that with a given finite nose length $l$, reducing the tip radius beyond $y_{0}$ corresponding to $y_{0}^{\prime}=0.7862$ actually has an adverse effect. To see this point, it is best to

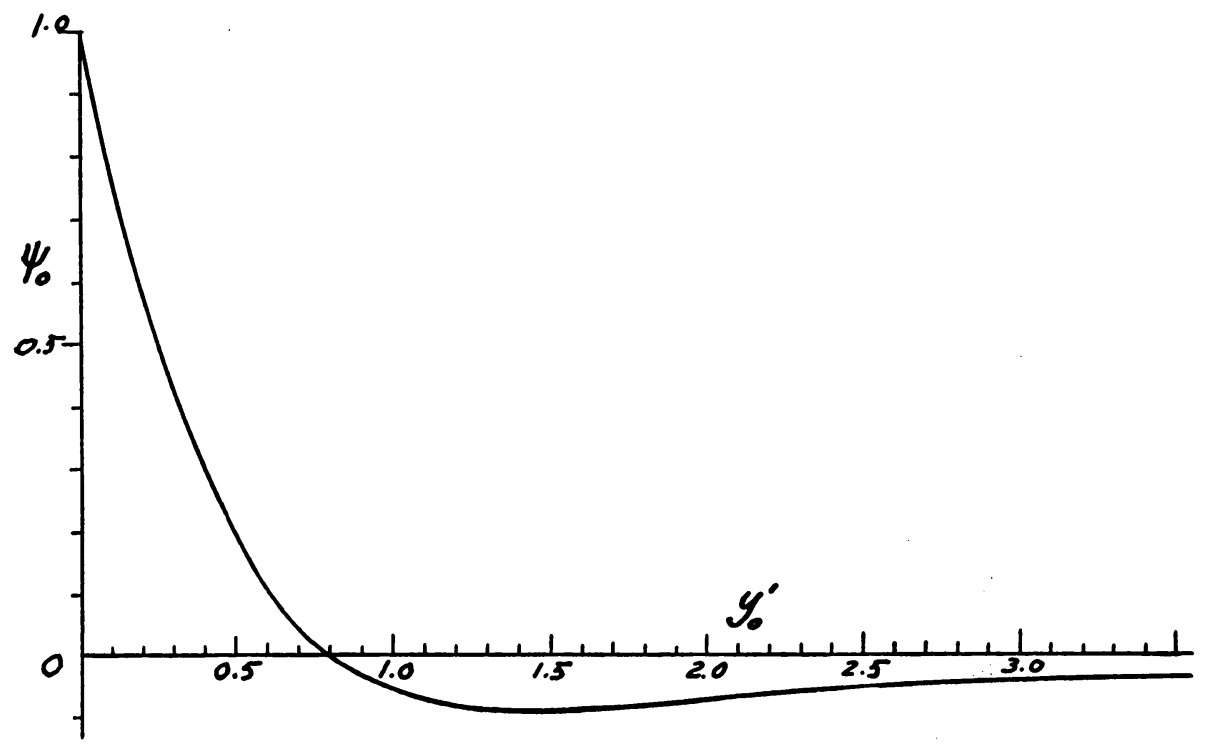

FIG. 1. 
refer to Fig. 1, which shows clearly that both for $y_{0}\left(y_{0}^{\prime}\right)$ greater and smaller than $y_{0}(.7862)$, we will have $\psi_{0} \delta y_{0}>0$, i.e., $\delta D>0$.

\section{REFERENCES}

1. G. A. Bliss, Lectures on the calculus of variations, University of Chicago Press, 1947

2. O. Bolza, Lectures on the calculus of variations, University of Chicago Press, 1904

3. G. A. Bliss, Calculus of variations, Open Court Co., 1925

4. H. and B. S. Jeffreys, Mathematical physics, Cambridge University Press, 1950

5. H. S. Tan, On superaerodynamic drag, and its minimization, to be published

6. H. S. Tan, On optimum nose curves for superaerodynamic missiles, J. Aeronaut. Sci. 25, 263 (1958)

\section{NOTE ON THE SOLUTION OF THE NEUTRON DIFFUSION PROBLEM BY AN IMPLICIT NUMERICAL METHOD*}

By GEORGE A. BAKER, JR. (Los Alamos Scientific Laboratory, Los Alamos, New Mexico)

It is of interest that the implicit, numerical method of Baker and Oliphant [1, 2] for the solution of time-dependent heat-flow problems in rectangular regions may be usefully extended to obtain the solution of the time-independent neutron-diffusion equation

$$
\nabla D(r) \nabla \varphi-\chi(r) \varphi+S(r)=0
$$

for the neutron density $\varphi$. In the original work of [1], to obtain an accurate solution, even asymptotically, it was necessary to take $\Delta t$ small. This choice was necessary because of the occurrence of a term in a higher order space derivative multiplied by $\Delta t$. We will herein make a slight modification to remove this defect. Let us rewrite (1) as (for the two-dimensional case)

$$
\begin{aligned}
\beta \varphi+\nabla^{2} \varphi+\frac{1}{\beta} \frac{\partial^{4} \varphi}{\partial x^{2} \partial y^{2}}=\alpha^{-2} \frac{\partial \varphi}{\partial t} & +\beta \varphi+\frac{1}{\beta} \frac{\partial^{4} \varphi^{*}}{\partial x^{2} \partial y^{2}} \\
& +\left(\frac{\chi(r)}{D(r)}-\mu\right) \varphi^{*}+\mu \varphi-\frac{S(r)}{D(r)}+\frac{\nabla D \cdot \nabla \varphi^{*}}{D(r)}
\end{aligned}
$$

where

$$
\varphi=\varphi^{*}, \quad \frac{\partial \varphi}{\partial t}=0
$$

and

$$
\mu=\operatorname{Min}_{r}[\chi(r) / D(r)] \text {. }
$$

To obtain the solution of (1) via (2) and (3) we must advance the time until the asymptotic solution of (2) is obtained. In [1], we described for the special case, $D$, a constant, how to guess $\varphi^{*}$, and then use (2) to calculate $\varphi_{\text {calc }}$. by solving the left-hand side and then how to compute a new guess, $\varphi^{* *}$, by means of

*Received September 30, 1958. Work performed under the auspices of the United States Atomic Energy Commission. 